\title{
Eight cases of sudden-onset dropped head syndrome: patient series
}

\author{
Kenji Endo, MD, PhD, Yasunobu Sawaji, PhD, Takato Aihara, MD, PhD, Hidekazu Suzuki, MD, PhD, Kazuma Murata, MD, PhD, \\ Yuji Matsuoka, MD, PhD, Hirosuke Nishimura, MD, PhD, Taichiro Takamatsu, MD, PhD, Takamitsu Konishi, MD, and \\ Kengo Yamamoto, MD, PhD
}

Department of Orthopedic Surgery, Tokyo Medical University, Tokyo, Japan

BACKGROUND As the proportion of elderly people continues to increase, the number of patients with dropped head syndrome (DHS) also grows. However, the relationship between onset and clinical course of DHS has hardly been studied, particularly, that of sudden-onset DHS has not been reported and remains unclear.

OBSERVATIONS Sudden-onset DHS was defined as presenting with chin on chest deformity within 3 days from the time of awareness of cervical weakness. Sixty-six patients with DHS visited our facility. Among them, 8 of the total cases (12.1\%) had experienced sudden onset DHS (6 females and 2 males; average age: $71.9 \pm 10.9$ years). Six of 8 cases showed recovery by conservative treatment, whose first interventions were from 0.1 to 12 months, but 3 experienced recurrence. Diffuse spinal kyphotic-type DHS was seen in 2 cases, and both had recurring horizontal gaze disturbance after initial recovery. Two unimproved cases underwent surgery of combined anterior and posterior cervical fixation, and their first interventions were at 5 and 24 months. After surgery, cervical sagittal alignment was improved, and they could walk maintaining horizontal gaze.

LESSONS Sudden-onset DHS can be expected to have a better outcome, but recurrence is possible in global imbalanced-type DHS.

https://thejns.org/doi/abs/10.3171/CASE21177

KEYWORDS dropped head syndrome; magnetic resonance imaging; histology; echography; sudden onset

The development of dropped head syndrome (DHS) is followed by severe cervical extensor muscle weakness, resulting in head overflexion or chin-on-chest deformity in the standing or sitting upright position. ${ }^{1}$ Isolated neck extensor myopathy (INEM) is 1 type of DHS and has been described in patients with cervical extensor muscle weakness in the absence of neuromuscular disorders. ${ }^{2}$ The etiology of DHS due to INEM has a low prevalence, even in elderly female persons. ${ }^{1}$ However, as the proportion of elderly people continues to rise, the number of patients with DHS due to degenerative changes is likely to increase as well. ${ }^{3,4}$ Patients with DHS present with inability to maintain a horizontal gaze and occasionally with cervical pain. Some patients complain of difficulty eating without hand support because of cervical instability. A typical characteristic of patients with DHS is that the cervical kyphosis can be correctable by passive extension; ${ }^{1}$ these patients do not show a dropped head condition in the temporary neutral position. In contrast, cervical spondylotic kyphosis usually presents cervical rigidity, ${ }^{1}$ and this condition cannot be corrected by passive cervical extension with decreased cervical range of motion. This is because cervical instability in DHS is mostly not associated with cervical bone or joint deformity, but with weakness of cervical extensor muscles and ligaments. Therefore, the onset and course of DHS is different from cervical spondylotic kyphosis.

DHS was defined as follows: 1) the patient showed chin-onchest deformity clinically after a few minutes in natural position, and 2) the deformity was correctable in the supine position. Correction of the cervical spine was confirmed by cervical MRI in the supine position. ${ }^{3}$ Sudden-onset DHS was defined as presenting with chin on chest deformity within 3 days from the time of being aware of cervical weakness. All subjects were examined by neurologists

ABBREVIATIONS DHS = dropped head syndrome; INEM = isolated neck extensor myopathy; $\mathrm{MRI}$ = magnetic resonance imaging; STIR = short-time inversion recovery.

INCLUDE WHEN CITING Published November 29, 2021; DOI: 10.3171/CASE21177.

SUBMITTED March 24, 2021. ACCEPTED August 10, 2021.

(c) 2021 The authors, CC BY-NC-ND 4.0 (http://creativecommons.org/licenses/by-nc-nd/4.0/). 
concerning possible Parkinson's or neuromuscular disease, and then diagnosed as INEM. Patients with neuromuscular disease and a history of previous spinal operation were excluded from this study. The onset of DHS could be classified into 2 types: ${ }^{5} 1$ acute within a few months and the other gradual over a few years. However, there is little evidence to date regarding the clinical course of DHS, and suddenonset DHS has never been reported. We experienced 8 cases of sudden-onset DHS due to INEM (6 females and 2 males; age: $71.9 \pm$ 10.9 years), and we herein present their backgrounds and outcomes.

Clinical details in cases are shown in Table 1, which presents clinical course, global alignment, associated pain, and outcome. The features of chin-on-chest deformity were examined by photographs or lateral cervical radiographs in an outpatient clinic. The primary treatment was conservative, consisting of cervical orthosis and physical therapy for at least 3 months for all cases. Cervical orthosis was recommended in order to maintain an upright position. Physical therapy was performed once a month at the outpatient clinic, and the patients practiced physical training daily at home. This physical training consisted of 2 types: 1 was global balance training, including the lumbopelvic region, and the other was isometric exercise to maintain an upright position. The mean follow-up period was 18.8 months ( 6 to 72 months). At the first visit to our facility, cervical and global radiographs and magnetic resonance imaging (MRI) were conducted. During the conservative treatment, some patients underwent pathological examinations by muscle biopsy or echography to analyze the muscle condition. Even after conservative treatment, surgical intervention was chosen for patients with continued severe quality-of-life disturbances. Regarding outcome, the improvement was defined by clinical evaluation (Table 2).

Regarding global spinal sagittal alignment, DHS can be divided into 2 types according to the Endo-Murata classification, ${ }^{6,7} 1$ being the cervical local kyphosis type that is maintained by compensatory function in the thoracolumbar spine (type $\mathrm{C}$ ), and the other the diffuse spinal kyphosis type that consists of the loss of global spinal compensatory function (type D). MRI was performed twice, once at the first visit and the second more than 6 months later. Cervical echography was conducted in 4 cases, and biopsy of the cervical extensor muscle in 3 cases at more than 3 months after the first visit.

\section{Study Description}

Sixty-six patients with DHS (14 men and 52 women; age: $74.0 \pm$ 9.7 years) visited our facility between January 2014 and March 2020. Among them, 8 of the total patients (12.1\%) had experienced sudden-onset DHS ( 6 females and 2 males; average age: $71.9 \pm$ 10.9 years).

\section{Clinical Findings}

Five cases (cases 1, 3, 6, 7, 8) presented with chin-on-chest deformity at waking up or within 24 hours, and their complaints were cervical pain and cervical weakness at waking up. Six cases (cases 1, 3, 4, 6, 7, 8) were accompanied by cervical pain at onset, which then lessened within 1 week. One case (case 5) presented with the deformity at 2 days after falling down. Three cases (cases $2,4,5)$ needed to support their chin by hand. All cases were treated with physiotherapy, together with the combination of a cervical brace, for at least 3 months. Six cases (cases 1, 2, 4, 5, 6, 8) showed improvement, and their first interventions were between 0.1 and 12 months (average: 3.6 months), although 3 cases (cases 2, $6,8)$ had recurrence (Tables 1 and 2). The two unimproved cases (cases 3,7$)$ underwent surgery of combined anterior and posterior

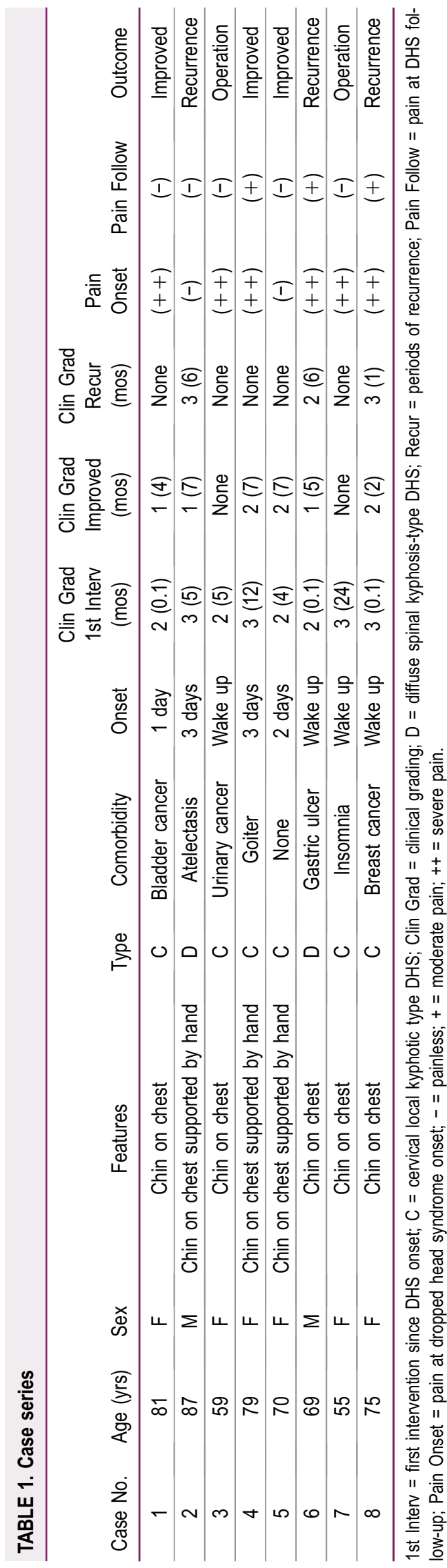


TABLE 2. DHS clinical grading

\begin{tabular}{cc}
\hline Grade & Description \\
\hline 0 & $\begin{array}{c}\text { Continuous gait possible more than } 30 \text { minutes without } \\
\text { cervical complain (normal condition) }\end{array}$ \\
\hline 1 & $\begin{array}{c}\text { Continuous gait possible more than } 30 \text { minutes with } \\
\text { cervical complain }\end{array}$ \\
\hline 2 & $\begin{array}{c}\text { Impossible to gait keeping horizontal gaze without } \\
\text { support more than } 30 \text { minutes }\end{array}$ \\
\hline 4 & $\begin{array}{c}\text { Impossible to gait keeping horizontal gaze without } \\
\text { support more than a few minutes }\end{array}$ \\
\hline$+\mathrm{N}$ & $\begin{array}{c}\text { Impossible to stand keeping horizontal gaze without } \\
\text { support more than a few minutes }\end{array}$ \\
\hline
\end{tabular}

Degree of improvement: 1 grade up; improve more than 2 grades up: well improved. Support means cane, walker, cervical, or trunk brace (excluding the reason without dropped head).

cervical fixation, and their first interventions were at 5 and 24 months. After surgery, their cervical sagittal alignment was improved, and they could walk maintaining horizontal gaze.

\section{Global Spinal Alignment}

Type C lobal sagittal alignment was seen in 6 (cases 1, 3, 4, 5, 7,8 ) and type $D$ in 2 (cases 2, 6) cases. Four cases (cases 2, 3, 4,6 ) also showed right-sided cervical scoliosis. The type $D$ (cases 2,6 ) cases (Table 1) had recurring horizontal gaze disturbance after initial recovery.

\section{Imaging Findings}

By short-time inversion recovery (STIR) MRI findings, high signal intensity was present at the cervical extensor muscle or interspinous space in 7 cases (cases 1, 2, 3, 4, 5, 7, 8), which then changed to isosignal intensity in 3 of the cases (cases 1, 4, 5). One case (case 6) showed high signal intensity at the intervertebral space and facet joint, which then had decreased 6 months later. Echography was performed in 4 cases (cases 2, 5, 7, 8) and pathological examinations in 3 cases (cases 2, 3, 8). At the elongated cervical interspinous space, Doppler echography revealed angiogenesis in all cases, and it was confirmed by pathological findings in 1 case (case 2).

\section{Illustrative Recurrence Case Presentation \\ Case 2}

An 87-year-old male complained of cervical pain and cervical weakness at the time of waking up, and within 3 days, he was having difficulty maintaining a horizontal gaze. His global sagittal spinal alignment was type D. His first visit was 5 months after onset (Grade 3), and the difficulty of horizontal gaze improved due to physiotherapy and a cervical brace fixation at 7 months after the first visit (Grade 1) (Fig. 1). However, 6 months after the improvement, his horizontal gaze disturbance worsened again (Grade 3). At 2 months after the recurrence, cervical plain radiography and echography showed elongation of the C6-7 interspinous and nuchal ligaments. At the C6-7 interspinous space, STIR MRI showed high signal change, and both echography and pathological findings showed angiogenesis (Fig. 2).
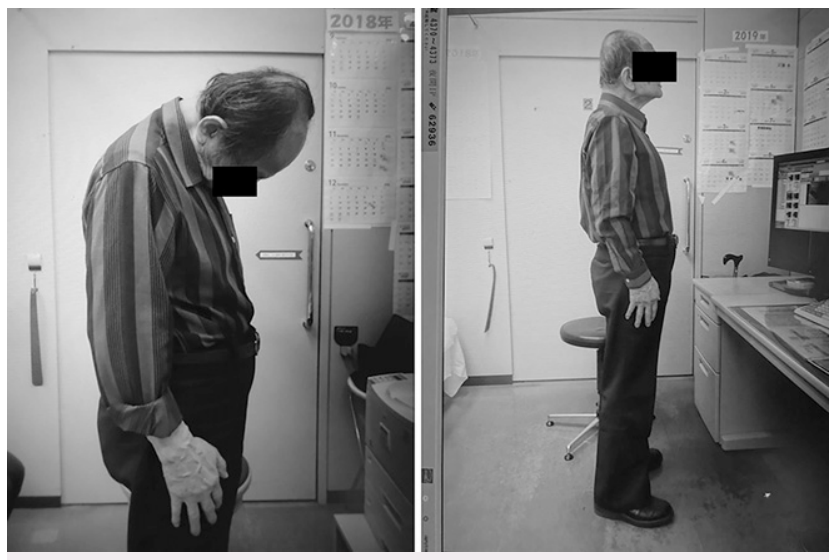

FIG. 1. Comparison of the posture at diagnosis and 7 months after our conservative treatment in case 2 .

\section{Discussion}

\section{Observations}

The present study showed a rate of sudden-onset DHS of $12.1 \%(8 / 66)$, and 6 of 8 cases (75\%) obtained clinical improvement, resulting in walking easier with horizontal gaze, but the recurrence rate was high $(50 \%)$. In 1996, Jaster et al. reported a case of natural recovery of myogenic cervical kyphosis (myogenic head drop) in 24 months. ${ }^{8}$ Previous literature mentioned that positive outcome by observation/bracing/physical therapy was $18.2 \%(6 / 33$ cases $)^{10}$ and $20.9 \%\left(14 / 67\right.$ cases). ${ }^{3}$ In the current study, 4 of 8 cases showed improvement in their horizontal gaze disturbance, and sudden-onset DHS had a better prognosis.

With respect to the onset of DHS, many cases were accompanied by cervical pain and hard-to-extend cervical spine at that time. After a few weeks, the cervical pain had decreased. Initial MRI findings showed high signal intensity in cervical extensor tissue in 7 of 8 cases, and follow-up MRI showed decreased signal intensity in 3 of the 7 cases. Previous literature has reported that fat-suppressed T2-weighted MRI showed diffuse muscular edema of the cervical extensor muscles. ${ }^{9,10}$ Echography and pathological findings during the chronic phase revealed the presence of angiogenesis in the elongated interspinous space. Pathological findings from muscle biopsy revealed fiber degeneration, regeneration, and necrosis with scattered inflammatory cells, which were similar to results of the previous report. ${ }^{8}$ Based on those findings, sudden-onset DHS is likely associated with acute cervical soft tissue damage and muscle weakness due to degenerative change..$^{11,12}$ In chronic phase, the regenerative process may occur, while causing angiogenesis, and some DHS cases could develop irreversible change caused by muscle fibrous degeneration.

The influence of cervical orthosis on the outcome was unclear because compliance varied depending on the cases. However, the present study does suggest that early intervention would be helpful for the improvement of DHS. The first interventions for the 2 unrecovered cases were delayed (4 and 24 months) in comparison with the recovered 6 cases (average: 3.6 months) (Table 1). Early intervention (physiotherapy, orthosis) can prevent recurrent hyperelongation of the extensor muscles. From the pathological findings, it was evident that damaged muscle fibers would finally be replaced by collagen fibers. At early periods after onset, posture training to stabilize the upright position is very important. If global spinal alignment could not be maintained, a worse prognosis would be expected. In the 

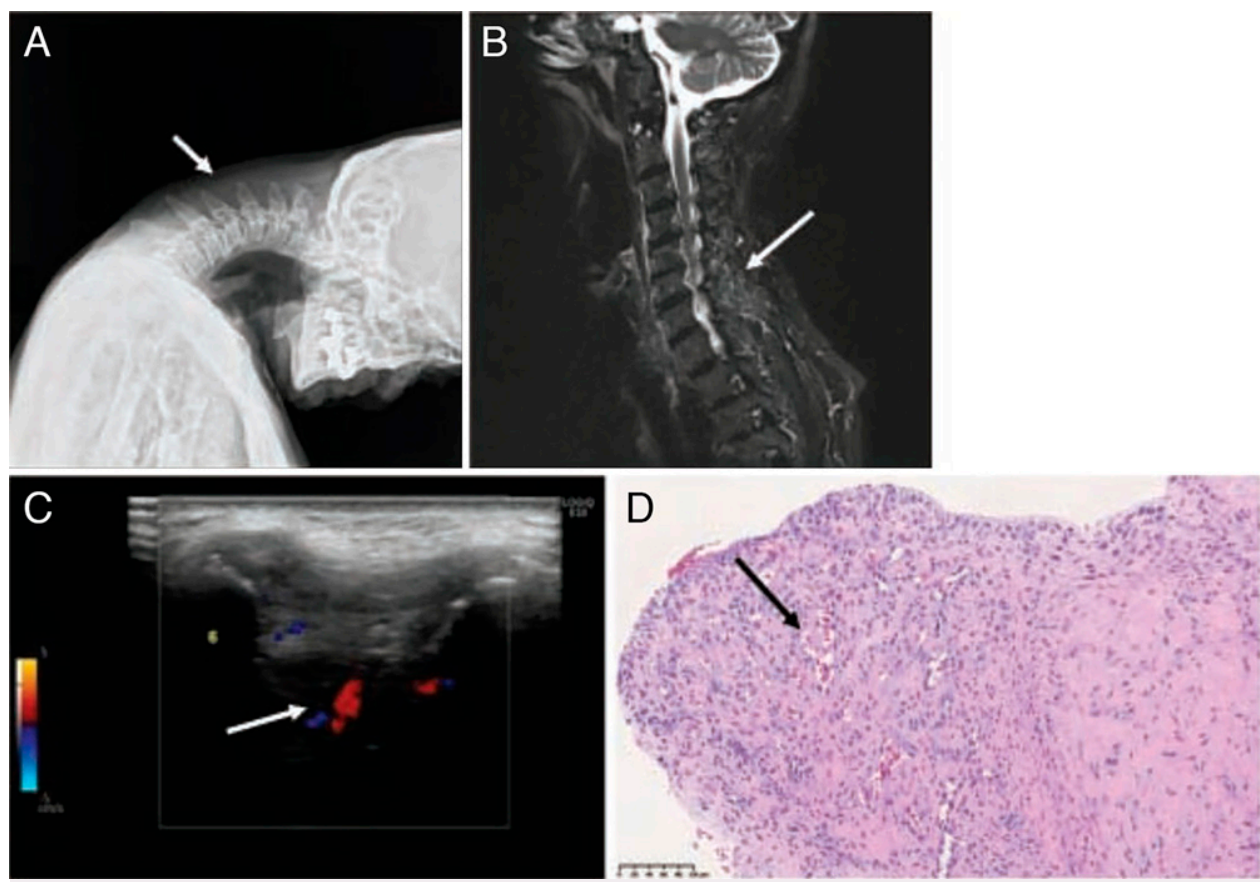

FIG. 2. Case 2. A: Lateral radiographic findings at cervical flexion (arrow indicates interspinous elongation between $\mathrm{C} 6$ and C7). B: Cervical sagittal MRI findings on STIR (arrow indicates signal change at cervical extensor muscles). C: Echography finding at interspinous space between C6 and C7 (arrow shows angiogenesis by Doppler method). D: Original magnification $\times 100$. Pathological findings at interspinous space between $\mathrm{C} 6$ and $\mathrm{C} 7$ (arrow indicates angiogenesis in interspinous ligaments).

present study, 2 cases needed to undergo surgery (cases 3,7 ). Because their first intervention was more than 4 months after onset, conservative treatment was not effective at all.

Regarding global spinal alignment, type $C$ was noted in 6 cases and type $D$ in 2 cases of sudden-onset DHS. The 2 type D DHS cases showed recurrence of horizontal gaze disturbance following initial improvement (Table 1). When DHS coincides with positive global imbalance (type D), physiotherapy should be included at the lumbopelvic region in addition to cervicothoracic exercise in order to regain their global spinal alignment. Global imbalance leads to concerns of causing excessive load to the cervical constructs, resulting in a possible recurrence of horizontal gaze disturbance. Even if the initial clinical findings showed improvement, it should be noted that the recurrence rate was high.

Examinations by cervical and global radiograph, MRI, and echography should be recommended initially and at 3 to 6 months after onset. Biopsy would be needed in cases of poor recovery. When surgical intervention is required, those findings will provide helpful information for establishing a prognosis based on conservative treatment.

\section{Lessons}

Sudden-onset DHS occurred in $12.1 \%$ (8/66) of DHS cases due to INEM. High signal change presented at the cervical extensor muscle in STIR MRI in the acute phase. Cervical interspinous spaces at the lower segments were elongated and showed angiogenesis in the chronic phase.

Better outcome can be expected by early intervention, but recurrence is possible in global imbalanced DHS.

\section{References}

1. Sharan AD, Kaye D, Charles Malveaux WM, Riew KD. Dropped head syndrome: etiology and management. J Am Acad Orthop Surg. 2012;20(12):766-774.

2. Katz JS, Wolfe GI, Burns DK, Bryan WW, Fleckenstein JL, Barohn RJ. Isolated neck extensor myopathy: a common cause of dropped head syndrome. Neurology. 1996;46(4):917-921.

3. Endo K, Kudo Y, Suzuki H, et al. Overview of dropped head syndrome (Combined survey report of three facilities). J Orthop Sci. 2019;24(6):1033-1036.

4. Brodell JD Jr, Sulovari A, Bernstein DN, et al. Dropped head syndrome: an update on etiology and surgical management. JBJS Rev. 2020;8(1):e0068.

5. Petheram TG, Hourigan PG, Emran IM, Weatherley CR. Dropped head syndrome: a case series and literature review. Spine (Phila Pa 1976). 2008;33(1):47-51.

6. Murata K, Endo K, Aihara T, et al. Relationship between cervical and global sagittal balance in patients with dropped head syndrome. Eur J Spine. 2020:29(3):413-419.

7. Endo K, Murata K, Suzuki H, Nishimura H, Tanaka H, Yamamoto K. The pathophysiology and classification of dropped head syndrome. Article in Japanese. Spine Spinal Cord. 2015;28(11):936-941.

8. Jaster JH, Bertorini TE, Swims MP, Dohan FC Jr, Menke PG, Becske T, Mönkemüller KE. Cervical kyphosis after resolution of myopathic head drop. A case report. Spine (Phila Pa 1976). 1996;21(17):2023-2025.

9. Gerling MC, Bohlman HH. Dropped head deformity due to cervical myopathy: surgical treatment outcomes and complications spanning twenty years. Spine (Phila Pa 1976). 2008;33(20): E739-E745.

10. Gaeta M, Mazziotti S, Toscano A, Rodolico C, Mazzeo A, Blandino A. "Dropped-head" syndrome due to isolated myositis of neck 
extensor muscles: MRI findings. Skeletal Radiol. 2006;35(2): $110-112$.

11. Eguchi $Y$, Toyoguchi T, Koda M, Suzuki M, Yamanaka H, Tamai H, et al. The influence of sarcopenia in dropped head syndrome in older women. Scoliosis Spinal Disord. 2017;12:5.

12. Murata K, Kenji E, Suzuki H, Takamatsu T, Nishimura H, Matsuoka Y, et al. Spinal Sagittal Alignment in Patients With Dropped Head Syndrome. Spine (Phila Pa 1976). 2018;43(21):E1267-E1273.

\section{Disclosures}

The authors report no conflict of interest concerning the materials or methods used in this study or the findings specified in this paper.

\section{Author Contributions}

Conception and design: Endo, Suzuki, Yamamoto. Acquisition of data: Endo, Murata, Matsuoka, Nishimura. Analysis and interpretation of data: Endo, Sawaji, Suzuki, Konishi. Drafting the article: Endo, Sawaji, Aihara, Murata, Takamatsu, Konishi. Critically revising the article: Endo, Aihara, Suzuki. Reviewed submitted version of manuscript: Endo, Sawaji, Suzuki, Murata, Konishi, Yamamoto. Approved the final version of the manuscript on behalf of all authors: Endo. Statistical analysis: Endo. Administrative/technical/material support: Endo, Sawaji. Study supervision: Endo, Yamamoto.

\section{Correspondence}

Kenji Endo: Tokyo Medical University, Tokyo, Japan. kendo@ tokyo-med.ac.jp. 